\title{
Dog ownership and contact during childhood and later allergy development
}

\author{
C-M. Chen, V. Morgenstern, W. Bischof, O. Herbarth, M. Borte, H. Behrendt, \\ U. Krämer, A. von Berg, D. Berdel, C.P. Bauer, S. Koletzko, H-E. Wichmann, \\ $J$. Heinrich and the Influences of Lifestyle Related Factors on the Human Immune \\ System and Development of Allergies in Children (LISA) Study Group and the \\ German Infant Nutrition Intervention Programme (GINI) Study Group
}

ABSTRACT: The effect of dog ownership during childhood on the development of allergy has been investigated in few studies with conflicting results. The association between dog contact and indoor endotoxin exposure during infancy and the development of allergic sensitisation and atopic disease up to age 6 yrs was investigated.

Two ongoing birth cohorts, the German Infant Nutrition Intervention Programme (GINI; $\mathrm{n}=1,962)$ and the Influences of Lifestyle Related Factors on the Human Immune System and Development of Allergies in Children (LISA; $n=1,193$ ), were analysed. In both studies, information on children's contact with dogs and their allergic symptoms and doctor-diagnosed allergic disease were collected during follow-up using questionnaires. Specific immunoglobulin $E$ to common aeroallergens was measured at age 6 yrs. House dust samples were collected at age 3 months and the amount of endotoxin was determined.

Dog ownership in early childhood was associated with a significantly lower rate of mixed pollen and inhalant sensitisation but not with dog sensitisation or allergic symptoms and diseases up to age 6 yrs. Regular contact with dogs, without ownership, during childhood was not associated with those health outcomes. No associations were found between house dust endotoxin exposure during infancy and sensitisation outcomes.

In conclusion, dog ownership in early childhood protects against the development of inhalant sensitisation and this effect cannot be attributed to the simultaneous exposure to endotoxin.

KEYWORDS: Allergy, cohort study, dog ownership, endotoxin, sensitisation

$\mathbf{T}$ he effect of pet ownership and pet contact on the development of allergic sensitisation and disease is disputed. Previous studies have mainly focused on cat allergen exposure and cat ownership, while not many prospective studies have specifically discussed the effect of childhood dog contact. Cohort studies have associated early childhood dog ownership with reduced risk of wheezing [1-3] and ALMQVist et al. [4] have reported that dog ownership in infancy seems to reduce the risk of asthma. Conversely, the Dutch Prevention and Incidence of Asthma and Mite Allergy cohort has reported that infancy dog allergen exposure has no effect on the development of wheezing or asthma [5]. Some cross-sectional studies have collected retrospective information on dog ownership in early childhood. In the European Community Respiratory Health Survey, SvANES et al. [6] reported that childhood dog ownership is associated with decreased risk of hay fever but promotes nonallergic asthma. Associations between childhood dog ownership and allergic sensitisation have also been reported [4, 7-9]. While most of the studies have observed a negative association between childhood dog ownership and sensitisation to aeroallergens, particularly outdoor aeroallergens, the Tucson birth cohort [3] reported that no such protective effect was found. The observed protective effect of dog ownership, however, may be partly due to selective dog avoidance by atopic parents [1012]. Conversely, the simultaneous exposure to higher level of indoor endotoxin may be the biological mechanism behind the observed protective effect $[13,14]$.

Higher level of endotoxin exposure in early childhood has been inversely associated with the development of allergic sensitisation and disease [15-17]. Studies have shown that endotoxin can stimulate the production of the cytokine

\section{AFFILIATIONS}

For affiliations, please see the Acknowledgements section.

CORRESPONDENCE

J. Heinrich

Institute of Epidemiology Helmholtz Zentrum Munchen German Research Center for Environmental Health Ingolstaedter Landstrasse 1 85764 Neuherberg Germany Fax: 498931873380 E-mail: joachim.heinrich@ helmholtz-muenchen.de

Received:

July 232007

Accepted after revision:

January 172008

\section{SUPPORT STATEMENT}

The present study was funded by grants of the Helmholtz Zentrum Munchen, German Research Center for Environmental Health (Munich), the Federal Ministry of Environment (BMU) for IUF (Institute for Environmental Research, Düsseldorf; grant FKZ 20462296) and the Federal Ministry for Education, Science,

Research and Technology (all Germany; grants no. 01EG9705/2 and 01EG9732).

STATEMENT OF INTEREST None declared. 
interleukin (IL)-12, which may consequently enhance young children's T-helper cell (Th) type 1 lymphocyte development and decrease their susceptibility for sensitisation to allergens [18-20]. Dog ownership has been directly associated with higher endotoxin level measured in settled house dust [1, 13, 14, 21, 22]. Therefore, it has been speculated that dog ownership during early childhood may protect young children from developing allergic sensitisation and disease.

The aim of the present study was to investigate the association between dog ownership and contact with dogs outside the domestic area and the development of inhalant sensitisation and allergic diseases in children aged $\leqslant 6$ yrs using two cohort studies conducted in Germany. An additional aim was to investigate whether endotoxin exposure during infancy, which has previously been associated with dog ownership, contributes to the protective effect against allergy.

\section{METHODS}

\section{Study population}

The study population was composed of two ongoing birth cohorts conducted in Germany, the Influences of Lifestyle Related Factors on the Human Immune System and Development of Allergies in Childhood (LISA) study and the German Infant Nutritional Intervention Programme (GINI). Detailed descriptions of screening and recruitment have been reportedly previously $[23,24]$.

Briefly, in the GINI study, 5,991 newborns from the 16 maternity wards in Munich and Wesel, Germany, fulfilling the inclusion criteria were recruited in the study between September, 1995 and June, 1998. There were two subgroups in the GINI study: an interventional and a noninterventional study arm (observational cohort group). A total of 2,252 infants with at least one parent or sibling having a history of allergic disease agreed to participate in the randomised trial, which aimed to compare the effect of hydrolysed formula and conventional cow's-milk formula on the prevention of the development of allergic disease in high-risk children. Infants with no family history of allergic disease and those whose parents refused to participate in the trial were allocated in the noninterventional study arm. Children from both groups were followed up at the age of 1, 2, 3, 4 and 6 yrs.

In the LISA study, the parents of neonates admitted to maternity hospitals in Munich, Leipzig, Wesel and Bad Honnef, Germany, were contacted. A total of 3,097 neonates were recruited in the study between December, 1997 and January, 1999. LISA was designed as a population-based study and the participants were not pre-selected based on family history of allergic disease. House dust samples were collected in the Munich and Leipzig subgroups. The cohort was followed up at the age of 6, 12 and 18 months, and 2, 4, and 6 yrs. Both studies were approved by the local ethics committees.

\section{Questionnaire data}

In both studies, information on parental educational level and parental history of allergic disease was collected using selfadministered questionnaires at birth. Information on children's allergic symptoms, including doctor-diagnosed asthma, eczema, allergic rhinitis, dog ownership, contact with dogs outside the home and environmental tobacco smoking at home, were collected at each follow-up using self-administered questionnaires. The question "(In the past 6/12 months/2 yrs) did your child have regular contact with any of the following animals outside the house - dog?" was used to assess any regular contact with dogs in nondomestic settings at each follow-up until the children were 4-yrs old.

\section{Collection and analysis of blood samples}

Blood samples were collected from 1,962 (51\%) and 1,193 (50\%) children from the GINI and LISA studies, respectively, at age 6 yrs (fig. 1). Specific immunoglobulin (Ig)E antibodies to aeroallergens were measured using the radioallergosorbant fluoroenzyme immunoassay CAP system (Pharmacia, Freiburg, Germany). A screening test for sensitisation was used to detect specific IgE antibodies against inhalant allergens (SX1 allergen mix, containing timothy grass, rye, birch, mugwort, house-dust mite, cat, dog and moulds) in the serum. The children who tested positive with SX1 were tested for single specific allergens, including the dog allergen, Can f1. Sensitisation to mixed pollen allergens included timothy grass, rye, birch and mugwort. Sensitisation was defined as having a specific serum $\operatorname{IgE}$ titre $>0.35 \mathrm{kU} \cdot \mathrm{L}^{-1}$ to specific allergens.

\section{Collection of settled house dust samples and analysis of endotoxin}

House dust samples were collected from 2,166 families in the Munich and Leipzig group of the LISA study, when the children were 3 months old. Trained field workers carried out dust sampling based on a standardised operating procedure $[21,22]$. Independent dust samples from parents' and children's mattresses were taken by vacuuming $1 \mathrm{~m}^{2}$ of each mattress surface for 2 min using vacuum cleaners equipped with special nozzles (allergen mouthpiece; ALK-Abelló, Hørsholm, Denmark). The samples were stored at $-20^{\circ} \mathrm{C}$ until extraction to prevent bacteria growth. The dust samples and filter paper were extracted by incubation with $0.125 \mathrm{M}$ ammonium bicarbonate $\left(\mathrm{NH}_{4} \mathrm{HCO}_{3}\right)$ plus $0.05 \%$ (volume/ volume) Tween 20 for $2 \mathrm{~h}$ at room temperature under constant shaking, with an extraction ratio of $10-1 \%$ (weight/v), depending upon the amount of sampled dust. House dust endotoxin levels (in endotoxin units (EU)) were quantified using the kinetic Limulus Amebocyte Lysate assay (Bio Whittaker, Walkerville, MD, USA) with a $50 \mathrm{EU} \cdot \mathrm{g}^{-1}$ dust detection limit. Endotoxin levels were expressed as endotoxin load (amount of endotoxin per square unit) of sample surface and endotoxin concentration (amount of endotoxin per gram of sampled dust).

\section{Information on household density in living area}

Information on household density (i.e. number of households per unit area of land) was available in the Munich subgroups from the GINI and LISA cohorts [25]. Demographic information including the number of households in every postcode area in Bavaria, Germany was collected from Infas GEOdaten (Bonn, Germany). The data were updated in December, 2003. The household density of a 2,500-m radius zone was calculated for each residential address of the Munich subgroups from both cohorts. The proportions of the postcode area and of the household counts were calculated for each zone and the 

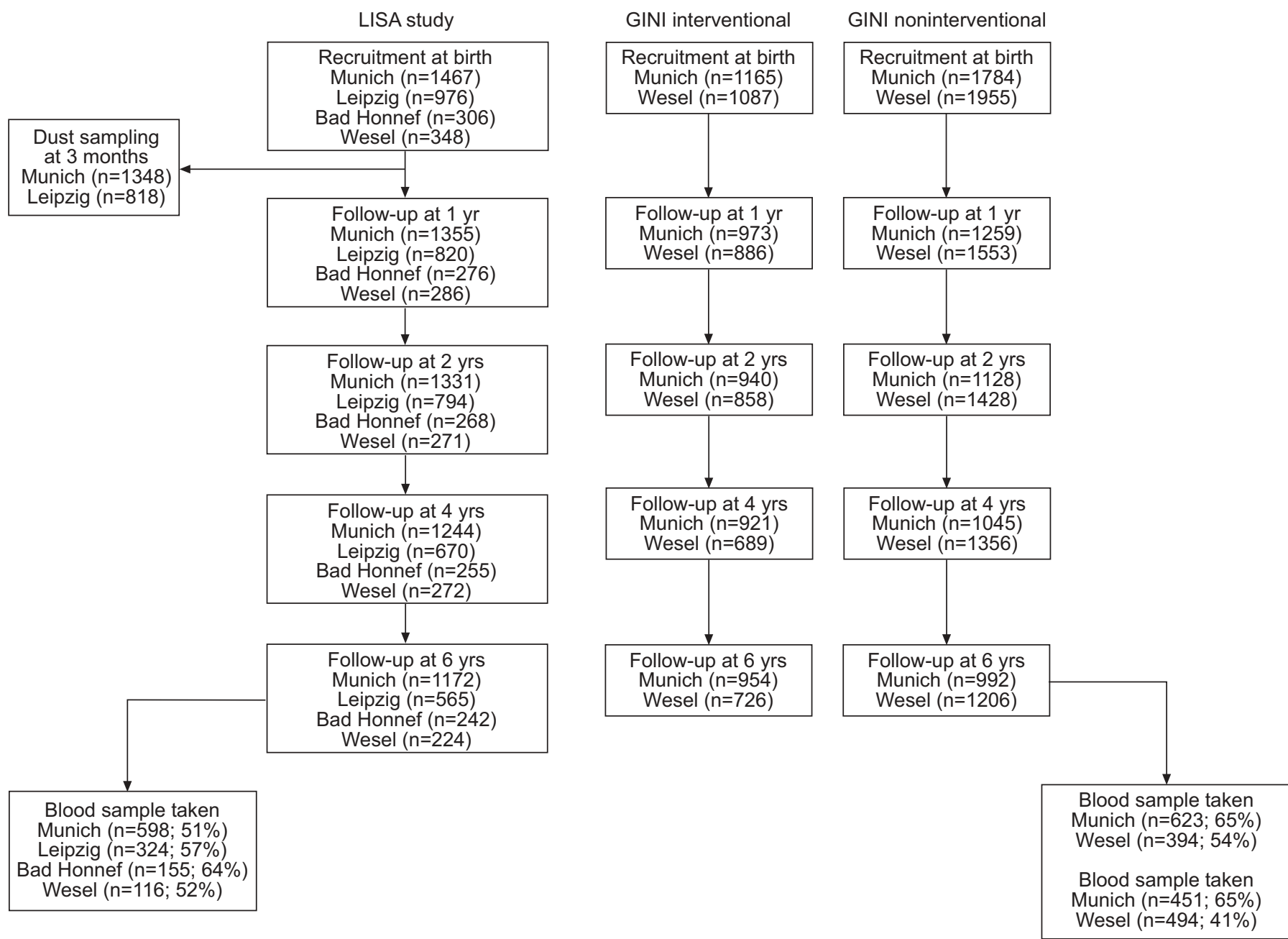

FIGURE 1. Consort diagram of the Influences of Lifestyle Related Factors on the Human Immune System and Development of Allergies in Childhood (LISA) study and the German Infant Nutritional Intervention Programme (GINI).

area-weighted averages of the household numbers were used as household density.

\section{Statistical analysis}

The correlation between endotoxin loads and concentrations from parents' and children's mattresses were calculated using Spearman correlation coefficient. Adjusted logistic regression models were fitted to describe the associations between dog ownership and regular dog contact outside the domestic area during childhood, and the development of dog, mixed pollen and inhalant sensitisation at age 6 yrs. To describe the association between dog ownership and contact, and the development of allergic symptoms and diseases between 4 $6 \mathrm{yrs}$ of age, longitudinal analysis with marginal logistic regression generalised estimating equation modelling was used. The longitudinal analysis approach takes the dependence of repeated outcome measures within each subject into account. In the statistical models, parental history of allergic diseases, parental education, sex and study centre were adjusted. In the sensitivity analyses, which assess the association between dog ownership and contact with dogs and the development of allergy taking cat ownership and contact with cats into account, cat ownership and contact with cats outside the domestic area were additionally adjusted. Endotoxin loads and concentrations in mattresses were normalised using logtransformation. An unpaired t-test was used in order to test whether log-transformed endotoxin loads and concentrations in mattresses were different between families with and without dog ownership. Adjusted logistic regression models were fitted in order to estimate the association between health outcomes and domestic endotoxin levels.

The results were presented as odds ratios with $95 \%$ confidence intervals. Wilcoxon rank-sum test was used to assess whether the household density is different between the dog owner and non-dog owner's living area.

\section{RESULTS}

Information regarding children's health outcomes and demographic characteristics is provided in table 1 . Due to the study design, only $26 \%$ of parents had a history of allergic disease in the GINI noninterventional study arm, as opposed to $90 \%$ in the GINI interventional study arm. The remaining $10 \%$ of the children in the GINI intervention study group had at least one sibling with a history of allergic disease. The high genetic risk 


\begin{tabular}{|c|c|c|c|c|}
\hline \multicolumn{5}{|l|}{ Sex } \\
\hline Male & 1586/3097 (51.2) & $1441 / 2814(51.2)$ & 1173/2252 (52.1) & $4200 / 8163(51.5)$ \\
\hline To dog allergen & 49/1193 (4.1) & 43/945 (4.6) & $64 / 1017(6.3)$ & $156 / 3155(4.9)$ \\
\hline To mixed pollen & 221/1193 (18.5) & 182/945 (19.3) & 238/1017 (23.4) & $641 / 3155(20.3)$ \\
\hline Inhalant allergens & $318 / 1193(26.7)$ & 257/945 (27.2) & 335/1017 (32.9) & $910 / 3155(28.8)$ \\
\hline \multicolumn{5}{|l|}{ Doctor diagnosed allergic disease } \\
\hline \multicolumn{5}{|l|}{ Eczema } \\
\hline Age 4 yrs & $56 / 2353(2.4)$ & $50 / 2378(2.1)$ & $66 / 1601(4.1)$ & $172 / 6332(2.7)$ \\
\hline Age 5 yrs & 90/2174 (4.1) & 66/2179 (3.0) & $120 / 1659(7.2)$ & 276/6012 (4.6) \\
\hline Age 6 yrs & $126 / 2165(5.8)$ & 77/2085 (3.7) & 137/1632 (8.4) & $340 / 5882(5.8)$ \\
\hline \multicolumn{5}{|l|}{ Asthma } \\
\hline Age 4 yrs & 23/2349 (1.0) & 20/2383 (0.8) & 32/1597 (2.0) & $75 / 6329(1.2)$ \\
\hline Age 5 yrs & 48/2173 (2.2) & $34 / 2176(1.6)$ & 49/1664 (2.9) & $131 / 6013(2.2)$ \\
\hline Age 6 yrs & $45 / 2169(2.1)$ & $34 / 2088(1.6)$ & 53/1634 (3.2) & $132 / 5891(2.2)$ \\
\hline \multicolumn{5}{|l|}{ Study centre (all Germany) } \\
\hline Munich & $1467 / 3097(47.4)$ & $1784 / 3739(47.7)$ & $1165 / 2252(51.7)$ & $4416 / 9088(48.6)$ \\
\hline Leipzig & $976 / 3097(31.5)$ & & & $976 / 9088(10.7)$ \\
\hline Wesel & $348 / 3097(11.2)$ & $1955 / 3739(52.3)$ & 1087/2252 (48.3) & 3390/9088 (37.3) \\
\hline
\end{tabular}

Data are presented as $\mathrm{n} / \mathrm{N}(\%)$, where $\mathrm{n}$ is the number of positive results and $\mathrm{N}$ is the total number tested. LISA: Influences of Lifestyle Related Factors on the Human Immune System and Development of Allergies in Childhood; GINI: German Infant Nutritional Intervention Programme. \#: categorised according to the German educational system as less than, equal to and more than grade 10 for high, medium and low, respectively; the highest education level from the parents was recorded.

of the children from the GINI intervention study group explains the higher sensitisation rates to dog, mixed pollen and inhalant allergens and the higher prevalence of diagnosed asthma, eczema and allergic rhinitis. The prevalence of dog ownership between birth and age 1 yr was $10 \%$ in the LISA cohort, $10 \%$ in the GINI noninterventional study arm and $8.5 \%$ in the GINI interventional study arm. At age 6 yrs, the prevalence of dog ownership was significantly different between the three study groups $(p=0.02)$. A total of $10 \%$ of the families in the LISA study had a dog, while 12 and $11 \%$ of the families in the GINI noninterventional and interventional study arms, respectively, owned dogs.

The associations between dog ownership or contact with dogs outside the domestic area versus the allergic sensitisation rate at age 6 yrs were investigated. Dog ownership in infancy, during early childhood and at age 6 yrs was not associated with dog sensitisation (table 2). However, dog ownership was associated with a significantly lower incidence of mixed pollen and inhalant allergen sensitisation, particularly if the child's family had a dog in the child's first year of life. Conversely, contact with dogs outside the domestic area without ownership was not associated with any of the sensitisation outcomes. Further investigation showed that dog ownership and contact with dogs outside the domestic area during childhood had no effect on the development of doctor-diagnosed allergic disease or reported allergic symptoms up to age 6 yrs (table 3 ).

In order to determine whether having contact with cats is an important confounder in the association between dog ownership and contact and the development of allergic sensitisation and diseases in young children, this was further investigated. Additional analyses were carried out with cat ownership and frequent cat contact outside the domestic area during childhood as two additional confounders. The results showed that adding the variables cat ownership and frequent cat contact 
TABLE 2 Dog ownership and contact during childhood and sensitisation to dog, mixed pollen and inhalant allergens at age 6 yrs ${ }^{\#}$

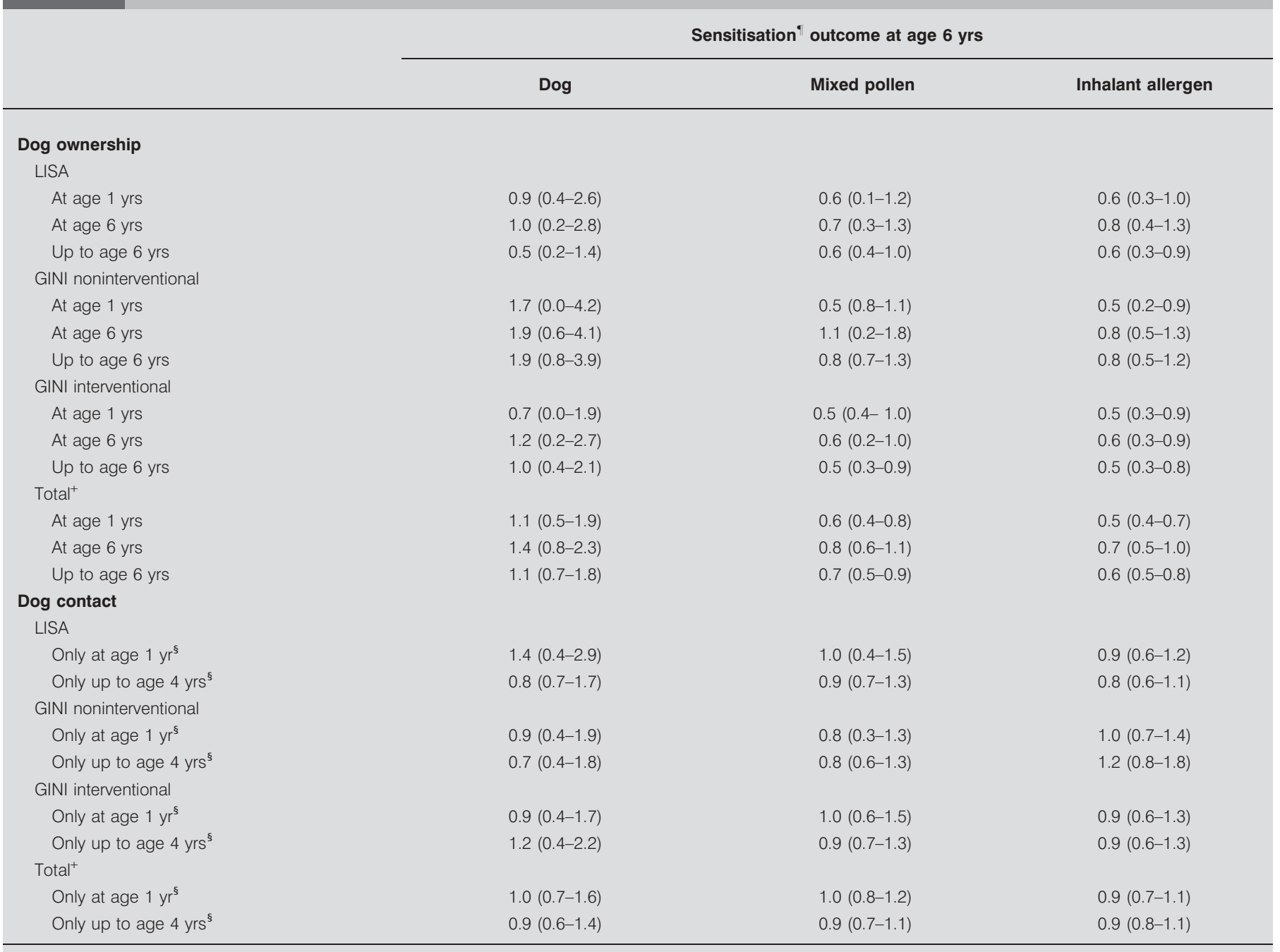

Data are presented as odds ratio (95\% confidence intervals). LISA: Influences of Lifestyle Related Factors on the Human Immune System and Development of Allergies in Childhood; GINI: German Infant Nutritional Intervention Programme. ": adjusted for sex, study centre, parental educational level and parental allergy; ": specific immunoglobulin E titre $>0.35 \mathrm{kU} \cdot \mathrm{L}^{-1}$ to tested allergen; ${ }^{+}$: additionally adjusted for studies; ${ }^{\varsigma}$ : dog owners were excluded.

outside the domestic area during childhood as the two additional confounders in the original models did not affect the estimated associations between dog ownership and contact during childhood and the development of allergic sensitisation in young children, in general, as well as in the sensitivity analysis, in which the children were stratified by parental history of allergic diseases (data not shown). The associations between dog ownership and contact during childhood, and the development of allergic diseases in young children were not strongly affected by the additionally added confounders of cat ownership and cat contact. Except for those children who had frequent contact with dogs in the first year of life in the GINI nonintervention study arm, it is now shown that these children have a statistically significantly higher probability of being diagnosed with asthma at school age. The probability of developing eczema symptoms in those children who have ever had a dog up to age 4 yrs in the GINI noninterventional arm is also shown to be higher (table 4).
Endotoxin samples were available from 2,108 mothers' mattress dust samples and 2,093 children's mattress dust samples from 2,166 families. All the dust samples analysed contained endotoxin above the detection limit. The median (25th-75th percentile) of the load and concentration of endotoxin from mothers' mattress dust were 2,071 (595$6,919) \mathrm{EU} \cdot \mathrm{m}^{-2}$ and $3,008(1,046-7,913) \mathrm{EU} \cdot \mathrm{g}^{-1}$, respectively, and in children's mattress dust samples, they were 1,015 $(330-3,022) \mathrm{EU} \cdot \mathrm{m}^{-2}$ and 5,866 $(2,336-14,669) \mathrm{EU} \cdot \mathrm{g}^{-1}$, respectively. The correlation between the amount of endotoxin in mothers' and children's mattress dust samples were 0.4 for both concentration and surface load. The amount of endotoxin in mothers' and children's mattress dust samples were significantly different between families with and without a dog in the household within the first year of the child's life $(\mathrm{p}<0.01)$. The median $(25 \mathrm{th}-75$ th percentile) of endotoxin load from mothers' mattress dust were 4,739 $(996-15,934) \mathrm{EU} \cdot \mathrm{m}^{-2}$ and 1,941 $(562-6,494) \mathrm{EU} \cdot \mathrm{m}^{-2}$ for families with and without 


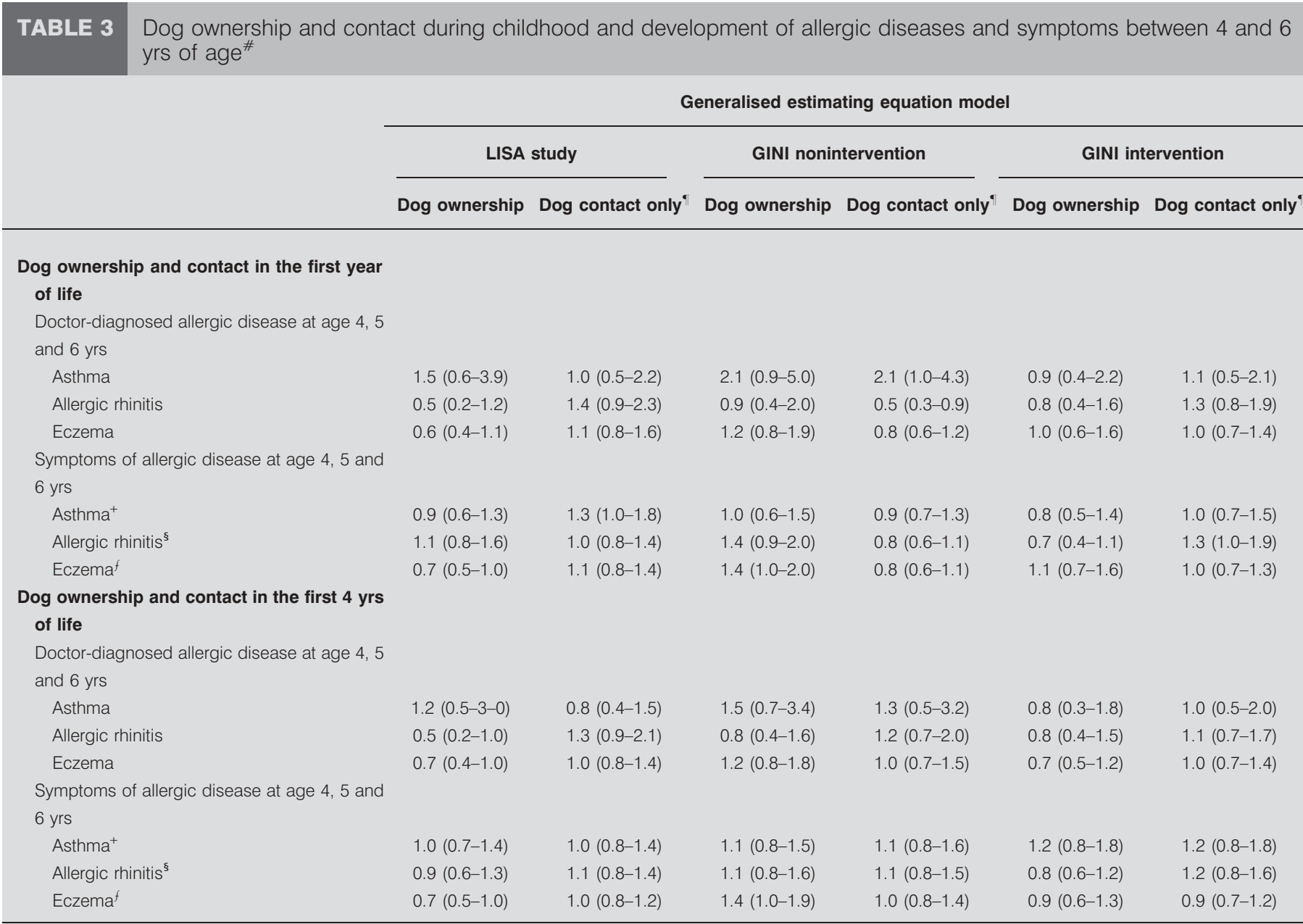

Data are presented as odds ratio (95\% confidence interval). LISA: Influences of Lifestyle Related Factors on the Human Immune System and Development of Allergies in Childhood; GINA: German Infant Nutritional Intervention Programme. ${ }^{*}$ : model adjusted for sex, study centre, parental educational level and parental allergy; ": dog owners were excluded; ${ }^{+}$: defined as wheezing sound in the chest; ${ }^{\text {s. }}$ : defined as sneezing, running nose or nasal congestion without cold; ${ }^{f}$ : defined as itchy rash that effected skin crease, face, neck, extremities, hands or feet.

dogs, respectively. For children's mattresses, the endotoxin loads were 1,724 (539-6,372) EU.m ${ }^{-2}$ and 949 (324$2,871) \mathrm{EU} \cdot \mathrm{m}^{-2}$ for families with and without dogs, respectively. However, further analysis showed that there were no associations between early childhood endotoxin exposure from mattresses, and sensitisation to: dog (odds ratio (OR; 95\% confidence intervals (CI)) 1.01 (0.80-1.26)); mixed pollen (1.07 (0.95-1.19)); and inhalant allergens (1.08 (0.97-1.19)). When using the endotoxin loads sampled from children's mattresses as exposure, similar results were observed with the amount of endotoxin sampled from parents' mattresses. The associations between endotoxin exposure and sensitisation outcome in homes with and without dog ownership in the child's first year of life were also assessed by including an interaction term of dog ownership in the first year and mattress endotoxin level in the model. The results showed that in homes with and without dog ownership, early childhood endotoxin exposure had no effect on the sensitisation outcomes at age 6 yrs (data not shown).

In a further subgroup analysis, it was investigated whether the household density where the dog owners live confounds the observed protective effect of dog ownership. In the Munich subgroups of both GINI and LISA cohorts, information on household density of the subjects' home addresses was available. It was found that dog owners were more likely to live in areas with lower household density $(\mathrm{p}<0.01)$. Further adjusting for household density in the model, however, did not modify the negative effect of dog ownership on sensitisation outcomes for pollen (0.53 (0.24-1.05)) and inhalant (0.51 (0.260.92)) sensitisation.

In order to overcome possible bias from selective avoidance of dog due to parental history of allergic disease, a stratified analysis was performed. The cohort was stratified by whether the parents had ever had asthma, eczema or hay fever and the association between dog ownership and contact, and the sensitisation rate at age 6 yrs was analysed again. The results confirmed that there was a negative association between dog ownership during childhood and sensitisation to mixed pollen and inhalant allergens at age 6 yrs. The negative associations were slightly stronger in families with a history of allergic disease (table 5). 
TABLE 4 Dog ownership and contact during childhood and development of allergic diseases and symptoms between 4 and 6 yrs of age, adjusted for cat ownership and frequent contact with cats

Generalised estimating equation model

\begin{tabular}{|c|c|c|c|c|c|}
\hline \multicolumn{2}{|c|}{ LISA study } & \multicolumn{2}{|c|}{ GINI nonintervention } & \multicolumn{2}{|c|}{ GINI intervention } \\
\hline Dog ownership & Dog contact only\# & Dog ownership & Dog contact only ${ }^{\#}$ & Dog ownership & Dog contact only \\
\hline
\end{tabular}

\begin{tabular}{|c|c|c|c|c|c|c|}
\hline \multicolumn{7}{|c|}{$\begin{array}{l}\text { Dog ownership and contact in } \\
\text { the first year of life }\end{array}$} \\
\hline \multicolumn{7}{|c|}{ Doctor-diagnosed allergic dis- } \\
\hline Allergic rhinitis & $0.5(0.2-1.3)$ & $1.5(0.9-2.4)$ & $0.9(0.4-2.0)$ & $0.5(0.3-0.9)$ & $0.7(0.3-1.4)$ & $1.3(0.8-2.0)$ \\
\hline Eczema & $0.7(0.4-1.2)$ & $1.1(0.8-1.6)$ & $1.2(0.8-2.0)$ & $0.8(0.5-1.2)$ & $1.0(0.6-1.6)$ & $0.9(0.6-1.3)$ \\
\hline \multicolumn{7}{|c|}{$\begin{array}{l}\text { Symptoms of allergic disease at } \\
\text { age } 4,5 \text { and } 6 \text { yrs }\end{array}$} \\
\hline Allergic rhinitis ${ }^{\S}$ & $1.1(0.8-1.7)$ & $1.1(0.8-1.5)$ & $1.4(0.9-2.0)$ & $0.9(0.6-1.2)$ & $0.6(0.4-1.0)$ & $1.4(1.0-1.9)$ \\
\hline Eczema $^{f}$ & $0.7(0.5-1.0)$ & $1.0(0.8-1.3)$ & $1.4(1.0-2.1)$ & $0.8(0.6-1.1)$ & $1.0(0.7-1.6)$ & $0.9(0.7-1.3)$ \\
\hline \multicolumn{7}{|c|}{$\begin{array}{l}\text { Dog ownership and contact in } \\
\text { the first } 4 \text { yrs of life } e^{\# \#}\end{array}$} \\
\hline \multicolumn{7}{|c|}{$\begin{array}{l}\text { Doctor diagnosed allergic dis- } \\
\text { ease at age } 4,5 \text { and } 6 \text { yrs }\end{array}$} \\
\hline \multicolumn{7}{|c|}{$\begin{array}{l}\text { Symptoms of allergic disease at } \\
\text { age } 4,5 \text { and } 6 \text { yrs }\end{array}$} \\
\hline Asthma $^{+}$ & $0.9(0.6-1.3)$ & $1.0(0.7-1.3)$ & $1.0(0.7-1.5)$ & $1.1(0.8-1.6)$ & $1.2(0.8-2.0)$ & $1.3(0.9-2.0)$ \\
\hline Allergic rhinitis ${ }^{\S}$ & $0.8(0.6-1.2)$ & $1.1(0.8-1.4)$ & $1.2(0.8-1.7)$ & $1.0(0.7-1.4)$ & $0.9(0.6-1.3)$ & $1.1(0.8-1.5)$ \\
\hline Eczema $^{f}$ & $0.7(0.5-1.0)$ & $1.1(0.8-1.3)$ & $1.5(1.1-2.1)$ & $1.1(0.8-1.5)$ & $0.8(0.5-1.2)$ & $0.9(0.6-1.2)$ \\
\hline
\end{tabular}

In order to overcome a potential confounding effect from exposure to environmental tobacco smoking, sensitivity analysis restricted to those children who have not been exposed to environmental tobacco smoke at home up to age 6 yrs $(n=1,752)$ was performed. The negative association between dog ownership during childhood and sensitisation to mixed pollen $(0.7(0.5-1.0))$ and inhalant allergens $(0.7$ (0.51.0)) at age 6 yrs remained.

Furthermore, in both the GINI and LISA studies, not all children agreed to participate in the blood examination. Therefore, the demographic characteristics between children with and without $\operatorname{IgE}$ measurements (table 6) were compared. It was found that parents with higher educational level were more likely to agree to participate in the $\operatorname{IgE}$ measurement, while parental history of allergic disease was negatively associated with participation in the $\operatorname{IgE}$ measurement. However, all children in the GINI interventional study arm are with at least one parent or sibling who has a history of allergic disease.

\section{DISCUSSION}

Dog ownership in early childhood was associated with a significantly lower rate of mixed pollen and inhalant sensitisation but not dog-specific allergic sensitisation. The negative associations remained when the data were stratified by parental history of allergic disease or restricted to the analysis of those children who had never been exposed to environmental tobacco smoke at home. Dog contact outside the domestic area alone had no effect on the development of allergic sensitisation. No consistent association between dog ownership and contact and the development of allergic symptoms and diseases was found. In homes with and without dog ownership, early childhood endotoxin exposure had no effect on sensitisation outcomes at age 6 yrs.

The protective effects of dog ownership in childhood on the development of inhalant allergen sensitisation in both low- and high-risk children have been reported $[4,6,7,26]$. Cumulative evidence suggests that dog ownership in early childhood prevents the development of allergy. Recently, however, a 


\begin{tabular}{|c|c|c|c|c|c|c|}
\hline \multirow{3}{*}{ TABLE 5} & \multicolumn{2}{|c|}{ Sensitisation to dog at age $6^{\circ}$} & \multicolumn{2}{|c|}{ Sensitisation to mixed pollen at age $6^{\circ}$} & \multicolumn{2}{|c|}{ Sensitisation to inhalant allergen at age $6^{\circ}$} \\
\hline & \multicolumn{2}{|c|}{ Parental allergy $^{+}$} & \multicolumn{2}{|c|}{ Parental allergy $^{+}$} & \multicolumn{2}{|c|}{ Parental allergy $^{+}$} \\
\hline & Yes & No & Yes & No & Yes & No \\
\hline At age $1 \mathrm{yr}$ & $0.7(0.2-1.5)$ & $1.7(0.6-4.0)$ & $0.5(0.3-0.8)$ & $0.6(0.3-1.1)$ & $0.4(0.3-0.7)$ & $0.6(0.3-1.0)$ \\
\hline At age 6 yrs & $1.3(0.6-2.4)$ & $1.7(0.7-3.8)$ & $0.6(0.4-1)$ & $1.0(0.6-1.6)$ & $0.6(0.4-0.9)$ & $0.8(0.5-1.2)$ \\
\hline Up to age 6 yrs & $0.9(0.4-1.6)$ & $1.6(0.7-3.5)$ & $0.5(0.3-0.8)$ & $0.8(0.5-1.2)$ & $0.5(0.4-0.8)$ & $0.7(0.5-1.0)$ \\
\hline \multicolumn{7}{|l|}{ Dog contact only ${ }^{\S}$} \\
\hline At age $1 \mathrm{yr}$ & $1.1(0.6-1.7)$ & $0.9(0.4-2.3)$ & $1.0(0.7-1.3)$ & $0.9(0.6-1.3)$ & $1.0(0.7-1.3)$ & $0.8(0.6-1.2)$ \\
\hline Up to age $4 \mathrm{yrs}$ & $1.0(0.6-1.6)$ & $0.7(0.3-1.7)$ & $0.9(0.7-1.2)$ & $0.9(0.6-1.4)$ & $0.9(0.7-1.2)$ & $0.9(0.7-1.4)$ \\
\hline
\end{tabular}

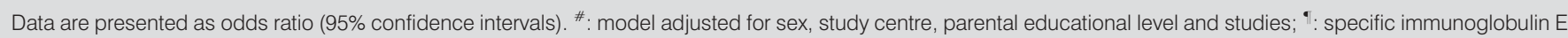
titre $>0.35 \mathrm{kU} \cdot \mathrm{L}^{-1}$ to tested allergen; ${ }^{+}$: defined as parent with asthma, eczema or hay fever; ${ }^{\S}$ : dog owners were excluded.

positive association between dog ownership in school-age children and the prevalence of dog sensitisation has been reported in a cross-sectional study in Kuwait. AL-MousAWI et al. [27] pointed out that the prevalence of dog ownership was extremely low in the study population $(1.5 \%)$, and so were the dog allergen levels in the children's home. Therefore, the result may not be suitable for generalisation to the communities

\begin{tabular}{|c|c|c|c|}
\hline \multirow[t]{2}{*}{ TABLE 6} & \multicolumn{3}{|c|}{$\begin{array}{l}\text { Demographic characteristics between children } \\
\text { with and without immunoglobulin (lg)E } \\
\text { measurements at age } 6 \text { yrs by study }\end{array}$} \\
\hline & & LISA & GINI \\
\hline \multicolumn{4}{|c|}{ Dog ownership at age 6} \\
\hline Yes & & 105/211 (50) & 216/446 (48) \\
\hline No & & $1083 / 1977$ (55) & $1739 / 3411(51)$ \\
\hline \multicolumn{4}{|c|}{$\begin{array}{l}\text { Parental history of asthma, } \\
\text { eczema or hay fever }\end{array}$} \\
\hline Yes & & $460 / 1272(36)^{*}$ & $766 / 2951(26)^{*}$ \\
\hline No & & 655/1585(41) & $1179 / 2946(40)$ \\
\hline \multicolumn{4}{|c|}{ Parental education level $^{\#}$} \\
\hline High & & $554 / 1263(44)^{*}$ & $757 / 1957(39)^{*}$ \\
\hline Medium & & 446/1191 (37) & 686/1930 (36) \\
\hline Low & & $1827606(30)$ & $517 / 2060(35)$ \\
\hline \multicolumn{4}{|c|}{$\begin{array}{l}\text { Environmental tobacco smoke } \\
\text { at home up to age } 6\end{array}$} \\
\hline Yes & & $396 / 1049(38)^{*}$ & $780 / 2021(39)^{*}$ \\
\hline No & & $762 / 1378(55)$ & $1080 / 2050(53)$ \\
\hline
\end{tabular}

Data are presented as $n / N(\%)$, where $n$ is the number of children who had $\lg E$ measurement at age 6 and $\mathrm{N}$ is the total number of children at age 6 yrs. LISA: Influences of Lifestyle Related Factors on the Human Immune System and Development of Allergies in Childhood; GINA: German Infant Nutritional Intervention Programme. ${ }^{*}$ : categorised according to the German educational system as less than, equal to and more than grade 10 for high, medium and low, respectively; the highest education level from the parents was recorded. *: $p<0.05$ from the Chi-squared test. where the prevalence of dog ownership is seven times higher. Simultaneous exposure to endotoxin has been speculated as the explanation behind the apparent protective effect, particularly due to a large number of studies reporting that children raised on farms, where high levels of endotoxin have been measured, have a lower prevalence of hay fever and allergic sensitisation [11, 16, 28, 29]. Laboratory experiments on mice have provided further evidence that endotoxin exposure induces cytokine production, which may shift the infants' developing immune system to predominantly Th1-type responses that protect children from developing allergy [19, 20]. GERN et al. [30] have also observed the association between dog ownership and higher IL-10 and IL-13 cytokine secretion in 1-yr-old children. However, the results from the present study and the cohort study in Boston [2] showed that the negative association between dog ownership and allergic sensitisation and symptoms are independent of the effect of endotoxin exposure.

It has also been speculated that the observed protective effect of dog ownership may be partly due to selective dog avoidance by atopic parents. It has been reported by the Swedish BAMSE (Children, Allergy, Milieu, Stockholm, Epidemiology) study [12] that dogs are less common in families with parental atopic eczema/dermatitis syndrome than in families without. However, the study has also reported that less dog avoidance behaviour was observed when compared with cat avoidance [12]. The recent publication from the European Community Respiratory Health Survey [31] reported that selective avoidance subsequent to asthma or allergy was not observed for childhood dog keeping and adult dog acquisition. In the present study, the negative association between dog ownership during childhood and sensitisation to mixed pollen and inhalant allergens at age 6 yrs was found in families with and without parental history of allergic disease. However, a stronger protective effect was observed in subjects with parental allergy. Since not all parents with a history of allergy also have a pet, especially dog, allergy, the possibility that dog avoidance may have partly contributed to the observed protective effect cannot be ruled out. 
In the present study, no associations between dog ownership and contact during childhood and the prevalence of allergic disease and symptoms between 4 and 6 yrs of age were found. In the previous publication of the GINI study, however, a negative association between keeping a dog in the child's first year of life and the development of eczema in the first and the second years of life was observed [23]. When children become older, they are more likely to have frequent contact with multiple triggers for allergic disease and symptoms, and the observed protective effect in infancy may, therefore, disappear when the children are of school age. In addition, it is possible that dog ownership during the child's first year of life is associated with a lower risk of early onset eczema, but that this association disappears at age $>2$ yrs. Additional adjustment for cat ownership and contact did not strongly affect the estimated associations with two exceptions. Within the GINI noninterventional study arm, children with frequent contact with dogs outside the domestic area are associated with a higher risk of being diagnosed with asthma, and those children who ever had a dog up to age 4 yrs seem to be more likely to have eczema symptoms. However, there are only 14, 27 and 26 children who have had frequent contact with dog at age $1 \mathrm{yr}$ and were diagnosed with asthma at age 4, 5 and 6 yrs, respectively. Furthermore, this estimated association was not echoed by the estimated associations in the LISA and GINI intervention study arm, nor was it echoed by the observed associations between dog ownership at age $1 \mathrm{yr}$ and the development of asthma in all three study populations. Therefore, this estimation might reach the statistical significance level by chance and a conclusion needs to be made cautiously. A similar argument can also be used for the estimated association between dog ownership up to age 4 yrs and the prevalence of reported symptoms of eczema in the GINI noninterventional arm. Further investigation for the associations between dog ownership and contact and the development of allergic diseases at an older age may provide more robust estimation. Finally, it is crucial to compare the effect of cat ownership and frequent contact with cats with the effect of dog ownership and frequent contact with dogs on the development of childhood allergy. The association between exposure to cat allergen and the development of allergy in children has been previously investigated in the Munich and Leipzig study populations of the LISA study [32]. It was observed that cumulative allergen exposure from cat ownership and regular cat contact during childhood increased the risk of cat sensitisation up to age 6 yrs. Exposure to cat allergen during infancy, however, was only associated with cat sensitisation at age 2 yrs. It was not associated with cat sensitisation at age 6 yrs or with allergic symptoms and diseases up to age 6 yrs. These two attempts to investigate the effect of exposure to pets on the development of allergy show substantial different effects from the two most popular family pets. Exposure to cat allergen during childhood directly increases the risk of developing cat sensitisation in children, while dog ownership during childhood is not associated with the development of $\mathrm{dog}$ sensitisation in children and it negatively associates with the development of inhalant sensitisation in children.

In the present study, it was not possible to establish how dog ownership leads to a lower atopy or how dog ownership was associated with a lower prevalence of mixed pollen and inhalant sensitisation but not with sensitisation to specific dog allergen. As the level of the dog allergen Can $\mathrm{f} 1$ in the house dust samples collected was not quantified, only the information on dog ownership as a surrogate of dog allergen exposure could be used. However, the observed protective effect is more likely due to other unknown factors associated with dog ownership. Dogs require more outdoor activities than most of the other pets. Dog fur is likely to carry a wide range of microbes other than endotoxin from the outdoor environment, such as soil. Close contact with dogs at a very young age may increase exposure to a variety of microbes and stimulate the maturation of the immune system. Keeping dogs also means a different lifestyle that involves more outdoor activities, which may explain why such protective effect was not observed in children who only had regular contact with dogs outside the domestic area but were not dog owners. Furthermore, some families may have had their dog before the birth of the child. It has been observed in laboratory experiments on mice that prenatal plus post-natal exposure to endotoxin is associated with a robust shift toward predominantly Th1 immune response [33]. This result corresponds to the finding that exposure to farm environment during pregnancy and the child's first year of life leads to a lower prevalence of allergy [34]. It has also been observed in the LISA study that exposure to high levels of endotoxin is negatively associated with cord blood IgE level [35]. Finally, OWNBY et al. [8] have reported that exposure to only one dog is not sufficient to stimulate children's immune system. Exposure to two or more dogs or cats in the first year of life is associated with a lower risk of inhalant sensitisation at age 7 yrs. Unfortunately, the information on the number of pets in each household of the present cohort was not available. Therefore, replication of the analysis by OWNBY et al. [8] was not possible. The fact that a greater number of indoor pets is associated with higher level of endotoxin may indicate that the amount of endotoxin exposure in most of the homes with only one $\operatorname{dog}$ is not sufficient to cause a modification in the development of children's immune systems. Conversely, the household density of the dog owners' homes had no effect on the negative associations between dog ownership and sensitisation outcomes.

One difficulty in the interpretation of the present results is that, as in every longitudinal cohort study, some participants were lost during follow-up and some did not participate in the IgE test at age 6 yrs. Although parental history of allergic disease was negatively associated with participation in the $\operatorname{IgE}$ measurement, the GINI noninterventional study arm, the LISA study population and the GINI interventional study arm represented low-, normal- and high-risk children respectively. Furthermore, there was no difference between dog ownership of participants and nonparticipants. However, the results should be interpreted with caution.

In conclusion, the present cohort studies provided evidence that dog ownership in early childhood protects against the development of inhalant sensitisation but not allergic symptoms and diseases up to age 6 yrs. It was also found that this protective effect could not be attributed to the simultaneous exposure to endotoxin or to avoidance measures. Further studies on the effect of a wider range of microbial exposure through dog ownership and pre-natal exposure are needed. 


\section{ACKNOWLEDGEMENTS}

The authors' affiliations are as follows. C-M. Chen, H-E. Wichmann: Institute of Medical Data Management, Biometrics and Epidemiology, Ludwig-Maximilians University of Munich; C-M. Chen, H-E. Wichmann, J. Heinrich, V. Morgenstern: Institute of Epidemiology, Helmholtz Zentrum Munchen, German Research Center for Environmental Health; C.P. Bauer: Clinic and Children's Clinic, Munich Technical University; S. Koletzko: Children's Hospital and Children's Clinic in Dr von Haunerschen Children's Hospital, Munich University Hospital; H. Behrendt: Helmholtz Zentrum Muchen, German Research Center for Environmental Health, and Dermatology and Allergology Clinic, Biederstein Technical University (all Munich, Germany). W. Bischof: Dept of Indoor Climatology, Institute of Occupational, Social, Environmental Medicine and Hygiene, Friedrich-Schiller University of Jena, Jena, Germany. O. Herbarth: Dept of Human Exposure Research and Epidemiology, Centre for Environmental Research Leipzig; and M. Borte: Dept of Child and Adolescent Medicine, St Georg Clinic, Academic Teaching Hospital, and Dept of Paediatrics, University of Leipzig (all Leipzig, Germany). U. Krämer: Institute for Environmental Research, Düsseldorf, Germany. A. von Berg, D. Berdel Marien: Hospital Wesel, Wesel, Germany.

The present authors would like to thank all families for their participation in the GINI and LISA studies.

\section{REFERENCES}

1 Campo P, Kalra HK, Levin L, et al. Influence of dog ownership and high endotoxin on wheezing and atopy during infancy. J Allergy Clin Immunol 2006; 118: 1271-1278.

2 Litonjua AA, Milton DK, Celedon JC, Ryan L, Weiss ST, Gold DR. A longitudinal analysis of wheezing in young children: the independent effects of early life exposure to house dust endotoxin, allergens, and pets. J Allergy Clin Immunol 2002; 110: 736-742.

3 Remes ST, Castro-Rodriguez JA, Holberg CJ, Martinez FD, Wright AL. Dog exposure in infancy decreases the subsequent risk of frequent wheeze but not of atopy. J Allergy Clin Immunol 2001; 108: 509-515.

4 Almqvist C, Egmar AC, Hedlin G, et al. Direct and indirect exposure to pets - risk of sensitization and asthma at 4 years in a birth cohort. Clin Exp Allergy 2003; 33: 1190-1197.

5 Brussee JE, Smit HA, van Strien RT, et al. Allergen exposure in infancy and the development of sensitization, wheeze, and asthma at 4 years. J Allergy Clin Immunol 2005; 115: 946-952.

6 Svanes C, Heinrich J, Jarvis D, et al. Pet-keeping in childhood and adult asthma and hay fever: European community respiratory health survey. J Allergy Clin Immunol 2003; 112: 289-300.

7 Hölscher B, Frye C, Wichmann HE, Heinrich J. Exposure to pets and allergies in children. Pediatr Allergy Immunol 2002; 13: 334-341.

8 Ownby DR, Johnson CC, Peterson EL. Exposure to dogs and cats in the first year of life and risk of allergic sensitization at 6 to 7 years of age. JAMA 2002; 288: 963-972.

9 Perzanowski MS, Rönmark E, Platts-Mills TA, Lundbäck B. Effect of cat and dog ownership on sensitization and development of asthma among preteenage children. Am J Respir Crit Care Med 2002; 166: 696-702.

10 Brunekreef B, Groot B, Hoek G. Pets, allergy and respiratory symptoms in children. Int J Epidemiol 1992; 21: 338-342.

11 Waser M, von Mutius E, Riedler J, et al. Exposure to pets, and the association with hay fever, asthma, and atopic sensitization in rural children. Allergy 2005; 60: 177-184.

12 Almqvist C, Egmar AC, van Hage-Hamsten M, et al. Heredity, pet ownership, and confounding control in a population-based birth cohort. J Allergy Clin Immunol 2003; 111: 800-806.

13 Heinrich J, Gehring U, Douwes J, et al. Pets and vermin are associated with high endotoxin levels in house dust. Clin Exp Allergy 2001; 31: 1839-1845.

14 Bischof W, Koch A, Gehring U, et al. Predictors of high endotoxin concentrations in the settled dust of German homes. Indoor Air 2002; 12: 2-9.

15 Williams LK, Ownby DR, Maliarik MJ, Johnson CC. The role of endotoxin and its receptors in allergic disease. Ann Allergy Asthma Immunol 2005; 94: 323-332.

16 Braun-Fahrländer C, Riedler J, Herz U, et al. Environmental exposure to endotoxin and its relation to asthma in schoolage children. N Engl J Med 2002; 347: 869-877.

17 Gehring U, Bischof W, Fahlbusch B, Wichmann HE, Heinrich J. House dust endotoxin and allergic sensitization in children. Am J Respir Crit Care Med 2002; 166: 939-944.

18 Lapa e Silva JR, Possebon da Silva MD, Lefort J, Vargaftig BB. Endotoxins, asthma, and allergic immune responses. Toxicology 2000; 152: 31-35.

19 Gerhold K, Blümchen K, Bock A, et al. Endotoxins prevent murine $\operatorname{IgE}$ production, $\mathrm{T}(\mathrm{H}) 2$ immune responses, and development of airway eosinophilia but not airway hyperreactivity. J Allergy Clin Immunol 2002; 110: 110-116.

20 Gerhold K, Bluemchen K, Franke A, Stock P, Hamelmann E. Exposure to endotoxin and allergen in early life and its effect on allergen sensitization in mice. J Allergy Clin Immunol 2003; 112: 389-396.

21 Platts-Mills JA, Custis NJ, Woodfolk JA, Platts-Mills TA. Airborne endotoxin in homes with domestic animals: implications for cat-specific tolerance. J Allergy Clin Immunol 2005; 116: 384-389.

22 Wickens K, Douwes J, Siebers R, et al. Determinants of endotoxin levels in carpets in New Zealand homes. Indoor Air 2003; 13: 128-135.

23 Zirngibl A, Franke K, Gehring U, et al. Exposure to pets and atopic dermatitis during the first two years of life. A cohort study. Pediatr Allergy Immunol 2002; 13: 394-401.

24 Zutavern A, Brockow I, Schaaf B, et al. Timing of solid food introduction in relation to atopic dermatitis and atopic sensitization: results from a prospective birth cohort study. Pediatrics 2006; 117: 401-411.

25 Morgenstern V, Zutavern A, Cyrys J, et al. Respiratory health and individual estimated exposure to traffic-related air pollutants in a cohort of young children. Occup Environ Med 2007; 64: 8-16.

26 Svanes C, Jarvis D, Chinn S, Burney P. Childhood environment and adult atopy: results from the European Community Respiratory Health Survey. J Allergy Clin Immunol 1999; 103: 415-420. 
27 Al-Mousawi MS, Lovel H, Behbehani N, Arifhodzic N, Woodcock A, Custovic A. Asthma and sensitization in a community with low indoor allergen levels and low pet-keeping frequency. J Allergy Clin Immunol 2004; 114: 1389-1394.

28 Schram D, Doekes G, Boeve M, et al. Bacterial and fungal components in house dust of farm children, Rudolf Steiner school children and reference children--the PARSIFAL Study. Allergy 2005; 60: 611-618.

29 von Mutius E. Environmental factors influencing the development and progression of pediatric asthma. J Allergy Clin Immunol 2002; 109: Suppl. 6, S525-S532.

30 Gern JE, Reardon CL, Hoffjan S, et al. Effects of dog ownership and genotype on immune development and atopy in infancy. J Allergy Clin Immunol 2004; 113: 307-314.

31 Svanes C, Zock JP, Antó J, et al. Do asthma and allergy influence subsequent pet keeping? An analysis of childhood and adulthood. J Allergy Clin Immunol 2006; 118: 691-698.

32 Chen CM, Rzehak P, Zutavern A, et al. Longitudinal study on cat allergen exposure and the development of allergy in young children. J Allergy Clin Immunol 2007; 119: 1148-1155.

33 Gerhold K, Avagyan A, Seib C, et al. Prenatal initiation of endotoxin airway exposure prevents subsequent allergeninduced sensitization and airway inflammation in mice. J Allergy Clin Immunol 2006; 118: 666-673.

34 Riedler J, Braun-Fahrländer C, Eder W, et al. Exposure to farming in early life and development of asthma and allergy: a cross-sectional survey. Lancet 2001; 358: 1129-1133.

35 Heinrich J, Bolte G, Hölscher B, et al. Allergens and endotoxin on mothers' mattresses and total immunoglobulin E in cord blood of neonates. Eur Respir J 2002; 20: 617-623. 